\title{
Positivity effect specific to older adults with subclinical memory impairment
}

\author{
Stephanie L. Leal, ${ }^{1,2,3}$ Jessica A. Noche, ${ }^{1,3}$ Elizabeth A. Murray, ${ }^{1}$ and Michael A. Yassa ${ }^{1}$ \\ ${ }^{1}$ Department of Neurobiology and Behavior, Center for the Neurobiology of Learning and Memory, Institute for Memory Impairments \\ and Neurological Disorders, University of California, Irvine, California 92697, USA; ${ }^{2}$ Department of Psychological and Brain Sciences, \\ Johns Hopkins University, Baltimore, Maryland 21218, USA
}

\begin{abstract}
Numerous studies have suggested that older adults preferentially remember positive information ("positivity effect"), however others have reported mixed results. One potential source of conflict is that aging is not a unitary phenomenon and individual differences exist. We modified a standard neuropsychological test to vary emotional content and tested memory at three time points (immediate/20 min/1 wk). Cognitively normal older adults were stratified into those with and without subclinical memory impairment. We found that the positivity effect was limited to those with subclinical memory impairment, suggesting that consideration of subclinical memory impairment is necessary for understanding age-related emotional memory alterations.
\end{abstract}

[Supplemental material is available for this article.]

Increasing age is the greatest risk factor for developing mild cognitive impairment (MCI) and Alzheimer's disease (AD) (Thies and Bleiler 2011). Thus, it is vital to understand neurocognitive changes that occur during the aging process to identify early phenotypes that may be associated with later cognitive decline. Memory impairment is one of the hallmark cognitive dysfunctions in healthy aging and is a major symptom of MCI and AD. There is extensive evidence that episodic memory, or memory for events, generally declines with age (Craik and Simon 1980; Glisky 2007). However, some studies suggest that there is a "positivity effect," where older adults may be more likely to attend to and remember positive information (Mather and Carstensen 2005; Wong et al. 2012). On the other hand, some studies have suggested that older adults are biased toward remembering negative details (Kensinger et al. 2007) or show no evidence of such an age-related positivity bias (Grühn et al. 2005; Fernandes et al. 2008). One potential source of conflict is that aging is not a uniform phenomenon and individual differences are frequently observed in older adults.

There are numerous approaches to examining individual differences with age. One such approach dichotomizes older rats into two groups (Gallagher et al. 1993). One group of older rats performs on par with young rats on the Morris Water Maze (i.e., "aged-unimpaired"-AU), while another group performs outside of the range of young rat performance (i.e., "aged-impaired"AI). This approach has been used to examine the aged brain in which this dichotomy maps onto neurobiological alterations associated with medial temporal lobe dysfunction, including synaptic loss in the perforant path (Smith et al. 2000), loss of inhibitory tone (Spiegel et al. 2013), hyperexcitability in the CA3 subregion of the hippocampus (Wilson et al. 2004, 2005), as well as loss of reelin and increased phosphorylated tau expression in the lateral entorhinal cortex (Stranahan et al. 2011). In humans, we have used a similar approach and have found that individual differences in age-related memory impairment (as defined by performance on the Rey Auditory Verbal Learning Test-RAVLT, a word-list

${ }^{3}$ These authors contributed equally to this work.

Corresponding author: myassa@uci.edu

Article is online at http://www.learnmem.org/cgi/doi/10.1101//m.042010.116. learning task) was associated with perforant path loss (Yassa et al. 2010), hyperactivation in the dentate/CA3 network (Yassa et al. 2011a), loss of entorhinal-hippocampal functional connectivity (Yassa et al. 2011b), and reduced dendritic integrity in the dentate/CA3 region (Yassa et al. 2011b). These cross-species findings suggest that this approach is fruitful and validated by neurobiological evidence in understanding individual differences in aging. Thus, we used the same approach here to divide older adults into two groups based on their performance on the RAVLT.

Thirty-two participants ( 21 female; mean age $74.8+4.4$ SD, range $=63-83$ ) were recruited from the local Orange County community via local campus announcements, flyers, and ads in local newspapers. Informed consent was obtained from all participants, with all procedures approved by the University of California, Irvine Institutional Review Board. All participants were screened against major medical or psychiatric morbidities as well as substance abuse history. All participants had normal or corrected-to-normal vision. Participants received a neuropsychological evaluation during their visit. The battery is shown in Table 1 . We then performed a median split, in which participants were divided based on their delayed recall performance on the RAVLT into AU $(N=16,11$ female; mean age $75.7+3.9 \mathrm{SD}$, range $=68-83$, RAVLT score $\geq 12)$ and AI $(N=16,10$ female; mean age $73.8+4.8 \mathrm{SD}$, range $=63-81$, RAVLT score $\leq 11)$. This method of splitting groups into AU and AI has been used previously (Stark et al. 2013; Reagh et al. 2014, 2015) and is in line with studies that show no differences between AU and young norms, while the AI group differs significantly from the young (Gallagher et al. 1993; Stark et al. 2010). Importantly, the AI group did not present with memory complaints, nor did they present with memory deficits sufficient for a diagnosis of clinical impairment. The particular selection of the RAVLT was motivated by the fact that it is a hippocampus-sensitive and a highly standardized

(C) 2016 Leal et al. This article is distributed exclusively by Cold Spring Harbor Laboratory Press for the first 12 months after the full-issue publication date (see http://learnmem.cshlp.org/site/misc/terms.xhtml). After 12 months, it is available under a Creative Commons License (Attribution-NonCommercial 4.0 International), as described at http://creativecommons.org/licenses/ by-nc/4.0/. 
Positivity effect and age-related memory impairment

Table 1. Demographic and neuropsychological test results in age-unimpaired (AU) and age-impaired (AI) older adults

\begin{tabular}{|c|c|c|c|c|}
\hline \multirow{2}{*}{$\begin{array}{l}\text { Groups } \\
\mathrm{N} \\
\text { Sex (M:F) } \\
\text { Age }\end{array}$} & \multicolumn{2}{|c|}{ Aged-unimpaired (AU) } & \multicolumn{2}{|c|}{ Aged-impaired (Al) } \\
\hline & \multicolumn{2}{|c|}{$\begin{array}{l}16 \\
5: 11\end{array}$} & & \\
\hline Variables & Mean & SEM & Mean & SEM \\
\hline Digit span forward & 9.8 & 0.5 & 9.8 & 0.6 \\
\hline Digit span backward & 6.7 & 0.5 & 6.0 & 0.5 \\
\hline Letter-number sequencing & 17.4 & 0.7 & 17.1 & 0.8 \\
\hline Geriatric depression scale & 0.8 & 0.4 & 1.6 & 0.4 \\
\hline Mini mental state exam & 28.6 & 0.3 & 27.9 & 0.4 \\
\hline RAVLT immediate recall & 13.1 & 0.3 & 8.3 & 0.5 \\
\hline RAVLT delayed recall & 13.1 & 0.3 & 7.9 & 0.6 \\
\hline RAVLT recognition recall & 14.3 & 0.2 & 12.5 & 0.5 \\
\hline Trail making test $\mathrm{A}$ & 31.6 & 2.0 & 29.0 & 2.3 \\
\hline Trail making test $\mathrm{B}$ & 78.7 & 5.9 & 82.8 & 9.2 \\
\hline Stroop test (word-color) & 30.4 & 2.4 & 30.5 & 1.8 \\
\hline Beck anxiety inventory & 3.8 & 1.4 & 6.0 & 1.3 \\
\hline Beck depression inventory-II & 3.8 & 0.8 & 3.1 & 0.7 \\
\hline Hours of sleep & 7.5 & 0.4 & 7.5 & 0.4 \\
\hline
\end{tabular}

Note: There were no significant differences between the groups in age, education, and all other neuropsychological measures $\left(P^{\prime} s>0.05\right)$ with the exception of RAVLT immediate recall $\left[t_{(30)}=-7.8, P<0.001\right]$, RAVLT delay recall $\left[t_{(30)}=-8.6, P<0.001\right]$, and RAVLT recognition $\left[t_{(30)}=-3.2, P=0.003\right]$.

neuropsychological test (Estévez-González et al. 2003). Furthermore, recent modeling work using the AD Neuroimaging Initiative suggests that changes in RAVLT performance are found very early in the clinical/pathological progression of $\mathrm{AD}$, even prior to detectable changes in amyloid pathology (Jedynak et al. 2012). We hypothesized that by examining older adults with and without subclinical memory impairment, we might be able to gain insight into the basis of the positivity effect and provide a more detailed assessment of emotional memory and recall over time in older adults.

To examine the impact of emotion on memory processing in older adults, we modified the logical memory subset (LMS) of the Wechsler memory scale III (WMS-III) to create the emotional logical memory test (ELMT), which includes one negative, one neutral, and one positive story, preserving all other aspects such as number of details and sentence structure. Each story had a word count between 60 and 70 words and was three sentences long. Each story was rated for valence and arousal on a scale by a separate group of participants (see results in Supplemental Information). After each story was verbally read to participants, they were instructed to repeat the story from memory as accurately as possible. Each story was read twice, and participants were asked to repeat as much as they could remember from the story after each reading (immediate recall, Imm). Responses were recorded and assessed for specific information (detail) and thematic information (gist) consistent with the WMS-III. There were a total of 25 possible points for detail information and 7 points for gist information for each story. The order of the stories was randomized across participants. Half of the neuropsychological battery was completed in the first session following the immediate condition of the ELMT. After 20 min of neuropsychological testing, participants were asked to recall as much as they could remember from each of the three stories (20-min delay, 20m). Responses were recorded as described above. Participants returned to the lab one week later, and were again asked to recall the three stories (1-wk delay, 1wk). The second half of the neuropsychological battery was then administered during the second visit (Fig. 1A). See Supplemental Information for the full stories used and characterizations of the stories in terms of arousal and valence (Supplemental Fig. S1 and Supplemental Methods). All statistical analyses were conducted in SPSS v. 23.0 (IBM Corp., released 2015,
Armonk, NY). Repeated-measures ANOVAs were corrected for error nonsphericity using Greenhouse-Geisser correction where appropriate. Post hoc statistical tests were conducted using trend analyses of planned comparisons and were corrected for multiple comparisons. Statistical values were considered significant at a final corrected $\alpha$ level of 0.05 , which appropriately controlled for Type I error.

We analyzed memory for gist and detail information (collapsing across emotion) by performing a two-way ANOVA with memory (gist and detail) and time (immediate-Imm, 20 min$20 \mathrm{~m}$, one week-1wk) as within-subjects factors. We found a significant effect of memory [gist $>$ detail; $F_{(1,31)}=257.04$, $P<0.001$; Fig. 1B] and a significant effect of time, $\left[F_{(2,62)}=\right.$ 90.62, $P<0.001]$, with worse memory for information over time $\left[F_{(1,31)}=111.01, P<0.001\right]$. We also found a significant interaction between memory and time $\left[F_{(2,62)}=10.69, P=0.001\right]$ with worse memory for detail information over time $\left[F_{(1,31)}=\right.$ 16.99, $P<0.001]$.

We calculated a forgetting rate for each participant (the difference between performance on the immediate and 1-wk delay tests) and found that there was more forgetting of detail information than gist information over 1 wk $\left[t_{(31)}=4.12, P<0.001\right.$; Fig. 1C]. Better memory (i.e., less forgetting) for the gist versus the details of a story as well as memory impairment over time are consistent with prior literature, suggesting that our task has external validity and can recapitulate known findings. While results from forgetting rate analyses amount to statistically the same results as performed above when including time as a factor, the forgetting rate measure allows us to determine forgetting on a subject-by-subject basis and allows for simpler visualization of the effects over time.

In a three-way ANOVA including emotion as a factor, we observed an additional significant effect of emotion [negative $>$ positive and neutral; $\left.F_{(2,62)}=34.15, P<0.001\right]$, and an interaction between emotion and memory $\left[F_{(2,62)}=32.22, P<0.001\right.$; Fig. $1 \mathrm{D}, \mathrm{E}]$. We further parsed this interaction and found that memory for negative gist information was superior to negative detail information, and this gist-detail relationship existed to a lesser extent for neutral and positive information $\left[F_{(1,31)}=\right.$ 55.73, $P<0.001]$. This suggests that while gist information is better remembered than detail information, the effect is greatest 
A

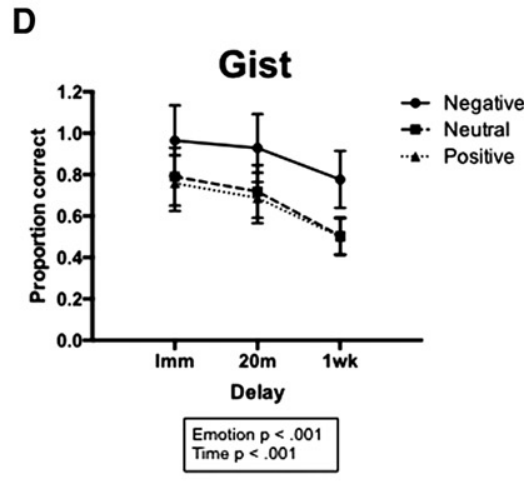

B

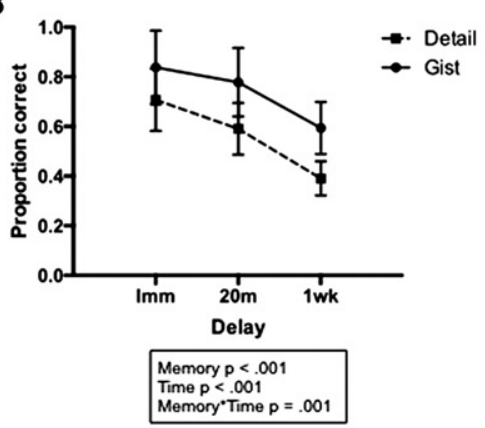

E

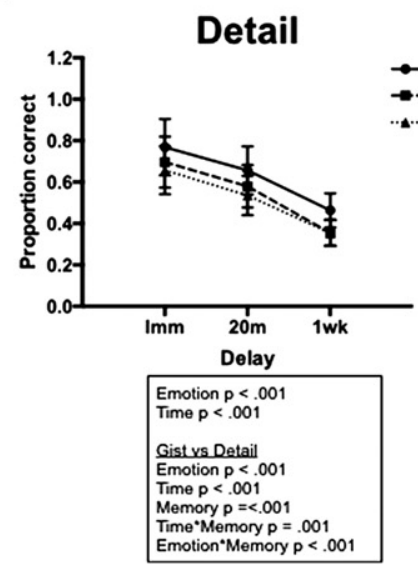

C

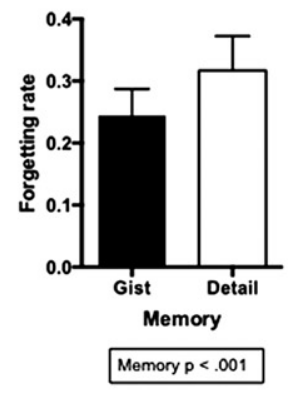

$\mathbf{F}$

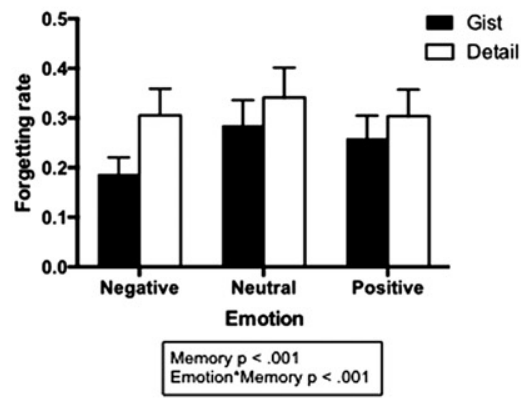

Figure 1. (A) Overall experimental schematic; $(B)$ overall memory performance (proportion correct) for gist and detail information in aging over time; (C) forgetting rate (immediate-1-wk delay) calculated within each subject for gist and detail information; $(D)$ proportion correct for gist information when split by emotional content (negative, neutral, and positive) in aging over time; (E) proportion correct for detail information when split by emotional content (negative, neutral, and positive) in aging over time; $(F)$ forgetting rate for gist and detail information split by emotional content.

when the information is negative compared with either positive or neutral information. In comparison of forgetting rates, we observed a significant interaction between emotion and memory $\left[F_{(2,62)}=3.44, P<0.001\right.$; Fig. $\left.1 F\right]$, with more forgetting of negative detail information compared with gist, which was not the case for positive and neutral gist information $\left[F_{(1,31)}=7.96, P=\right.$ 0.008]. This suggests that while negative information is better remembered overall compared with neutral and positive information, negative details are more likely to be forgotten after a 1-wk delay. These analyses strongly suggest that both the emotional content of information and the amount of time passed influence the fidelity of memory in older adults.

To assess how emotional memory is altered in the course of age-related cognitive decline, we grouped our participants based on performance on the RAVLT delayed recall test and created AU and AI groups using a median split (Gallagher et al. 1993; Stark et al. 2010). Here, we are only reporting significant effects or interactions with group, as we have already discussed the effects of emotion, memory, and time above, which were replicated in this analysis. In a three-way ANOVA with time and memory as within-subjects factors and group (AU and $\mathrm{AI}$ ) as a betweensubjects factor, we found a significant effect of group $\left[F_{(1,30)}=\right.$ 4.94, $P=0.03$; Fig. 2A,B], with worse memory in the AI group compared with AU. These findings are consistent with past work and with a generalized memory deficit in the AI group.

We then conducted separate three-way ANOVAs for gist and detail in order to examine the effect of emotion. For gist, we found a significant three-way interaction between emotion, time, and group $\left[F_{(4,120)}=4.52, P=0.004 ;\right.$ Fig. $\left.2 \mathrm{C}, \mathrm{D}\right]$, where the AI group had worse memory for neutral gist information over time, where- as the AU group had worse memory for positive gist information over time $\left[F_{(1,30)}=12.34, P=0.001\right]$. There was a marginal effect of group $\left[F_{(1,30)}=3.46, P=0.07\right]$, where the AI group showed worse memory for gist information compared with AU. For detail information, we observed a significant effect of group $\left[F_{(1,30)}=\right.$ 5.96, $P=0.02$; Fig. 2E,F], where the AI group showed worse memory for detail information compared with AU. We also found a marginal interaction between emotion, time, and group $\left[F_{(4,120)}=2.5, P=0.065\right]$, where the AI group had worse memory for neutral detail information over time compared with the AU group. This is a similar pattern to what we saw for remembering gist information, although it was not as statistically reliable. We also conducted a four-way ANOVA to directly compare memory, emotion, time, and group together. These results confirmed our findings from the separate three-way ANOVAs (see Supplemental Information for results).

In comparing forgetting rates for gist information, we found a significant interaction between emotion and group $\left[F_{(2,60)}=\right.$ 7.15, $P=0.002$; Fig. 3A]. The AI group exhibited the most forgetting of neutral information, whereas the AU group exhibited the most forgetting of positive information $\left[F_{(1,30)}=\right.$ $12.33, P<0.05]$. For detail information, we found a significant interaction between emotion and group $\left[F_{(2,60)}=3.28, P=0.045\right.$; Fig. 3B]. Similar to the results for gist information, the AI group exhibited less forgetting of positive and more forgetting of neutral detail information than the AU group $\left[F_{(1,30)}=5.74, P<0.05\right]$. In a direct comparison of gist and detail, we found a significant three-way interaction between emotion, memory, and group $\left[F_{(2,60)}=3.44, P=0.04\right]$. Post hoc contrasts showed that the effects noted above (AI exhibiting less forgetting of positive and 
A

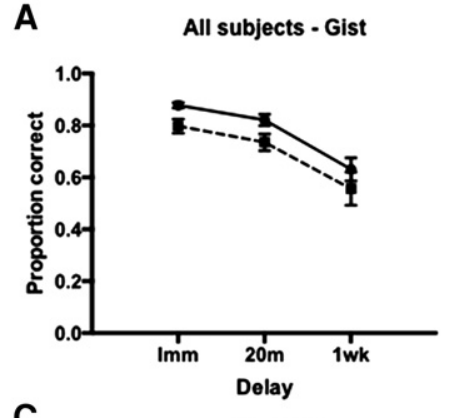

C

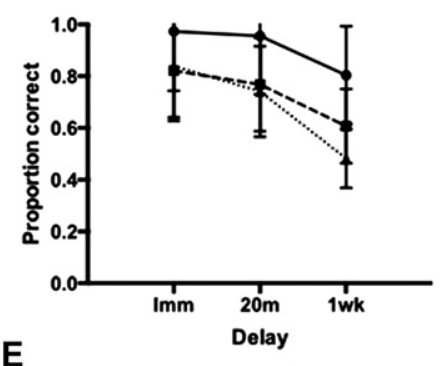

E

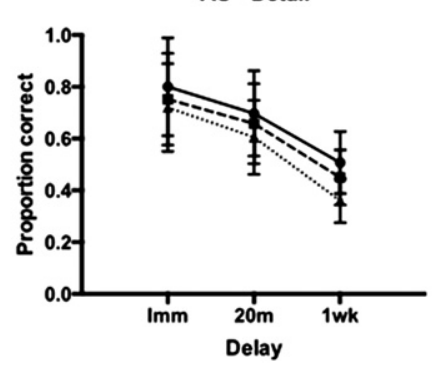

B

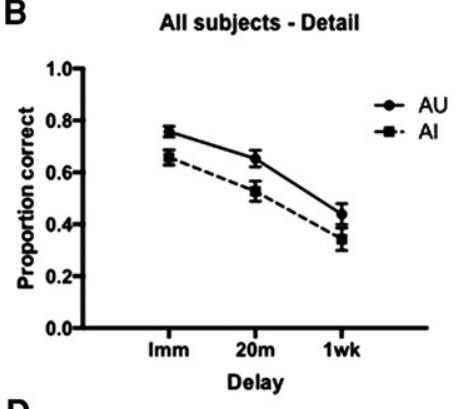

D
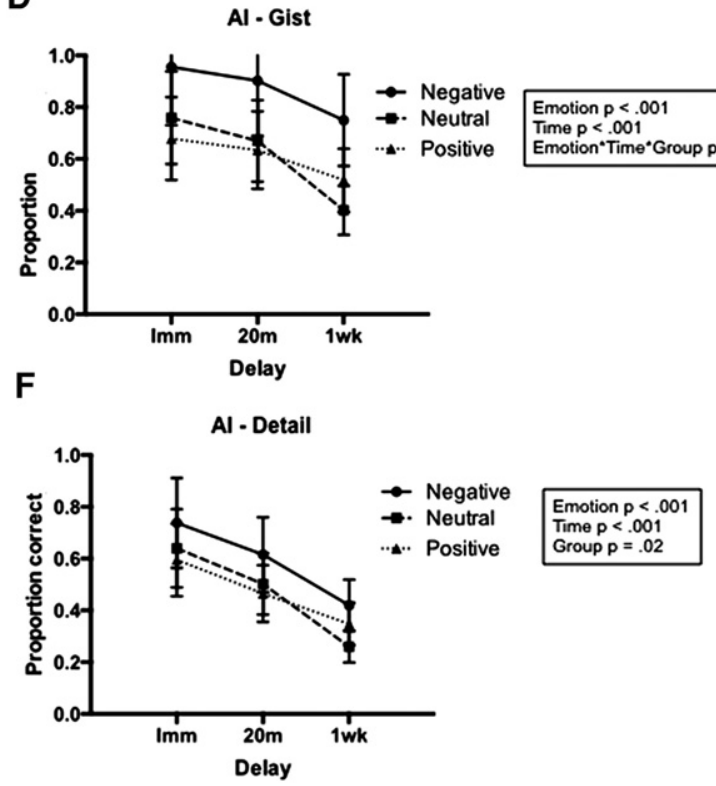

Time $p<.001$

Group $p=.03$

Group $p=.03$
Time Memory $p=.001$

Figure 2. Individual differences in memory performance. $(A)$ Gist and $(B)$ detail remembering in aged-unimpaired (AU) and aged-impaired (AI) over time; performance for gist information split by emotional content in $(C)$ AU versus $(D)$ Al adults; performance for detail information split by emotional content in $(E) \mathrm{AU}$ versus $(F)$ Al adults.

more forgetting of neutral information than AU) were larger for gist than detail information $\left[F_{(1,30)}=6.33, P=0.02\right]$.

To further investigate the relationship between emotional forgetting and subclinical memory impairment, we calculated a difference score of the forgetting rates for each subject to measure emotional relative to neutral performance (Positivity effect: Positive-Neutral forgetting rate and Negativity effect: Negative-Neutral forgetting rate). This was done for gist and detail information separately. We found that the positivity effect was positively correlated with RAVLT-delayed score such that lower RAVLT scores were associated with less positive forgetting while higher RAVLT scores were associated with more positive forgetting [gist: $r=0.499, P=0.006$, detail: $r=0.460, P=0.012$, both controlled for age, sex, and education; Fig. 4A,B]. This was not the case for negative information $\left(P^{\prime} \mathrm{s}>0.05\right)$ and further supports the relationship between positive memory and subclinical memory impairment.

Overall, these findings suggest that relative to healthy older adults, those with subclinical memory impairment (i.e., AI) are more prone to forgetting neutral rather than emotional information. However, healthy older adults (i.e., AU) are more prone to forgetting positive gist information. These effects are similar across gist and detail information but appear to be more prominent in gist memory.

While numerous neuropsychological assays have been used to assess memory impairments, emotional content has been large- ly ignored as a factor that can alter how memories are stored and recalled in neuropsychological tests. For example, the stories in the LMS of the WMS-III contain emotional and neutral content, but memory is assessed as a whole without consideration for emotion. This could yield an incomplete account of how memory is altered in aging and disease, since memory systems underlying emotional memory are complex and are altered in the context of age and neuropsychiatric disorders. In the current study, we
A

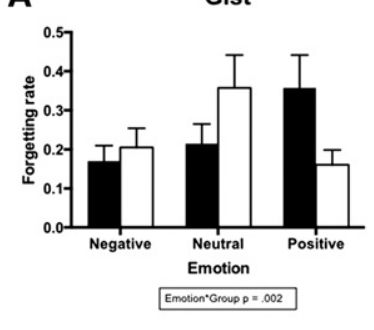

B Detail

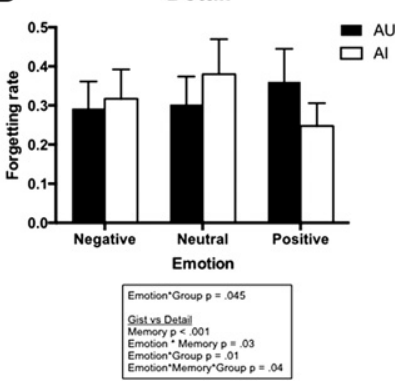

Figure 3. (A) Forgetting rate for gist information across aged-unimpaired $(A U)$ and aged-impaired (AI) split by emotional content, $(B)$ forgetting rate for detail information across aged-unimpaired $(\mathrm{AU})$ and aged-impaired (Al) split by emotional content. 
A

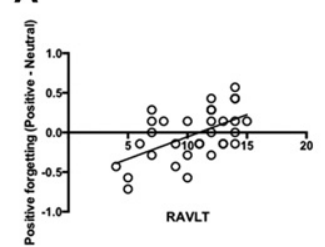

B

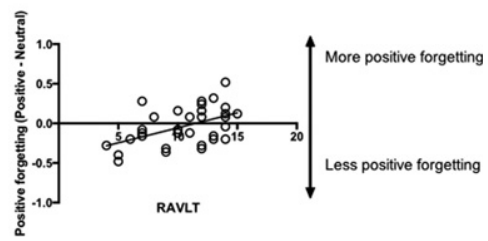

Figure 4. (A) Gist positive forgetting (Positive-Neutral) for each subject correlated with RAVLT-delayed test, controlled for age, sex, and education, $(B)$ detail positive forgetting (Positive-Neutral) for each subject correlated with RAVLT-delayed test, controlled for age, sex, and education.

created three stories that separately test negative, neutral, and positive content. We tested memory in healthy older adults across three different time points to better understand how emotional memory changes over time. We also investigated whether this task may be sensitive to subtle cognitive deficits by examining how individual differences in memory performance were associated with emotional memory processing.

We found that gist information was remembered better than detail information in older adults overall. This is consistent with previous findings suggesting that aging is associated with decreased recollection and preserved familiarity, and also fits with computational approaches suggesting that older adults are more biased toward memory generalization at the expense of discrimination (Yassa and Stark 2011; Koen and Yonelinas 2014). However, we did not compare our sample to young adults, so it is unknown whether AU adults show similar performance to that of young adults. When analyzing performance based on the emotional content of the stories, we found that negative information was selectively preserved compared with neutral or positive information. Preferentially remembering positive information in older adults (i.e., the positivity effect) has been documented in the literature (Mather and Carstensen 2005), although other studies have suggested that older adults preferentially remember negative information (Kensinger 2008). We hypothesized that this discrepancy may be resolved by using an individual differences approach to testing memory in older adults. Our analyses of individual differences yielded several interesting results. We found that $\mathrm{AI}$ individuals had better memory (i.e., less forgetting) for positive information and worse memory (i.e., more forgetting) for neutral information compared with AU individuals. Furthermore, lower RAVLT scores were associated with more neutral relative to positive forgetting while higher RAVLT scores were associated with more positive relative to neutral forgetting. This difference between AU and AI groups suggests that the positivity bias previously reported in aging may be driven at least in part by subclinical memory impairment. Interestingly, it has been proposed that the positivity effect seen in aging occurs to a larger extent in those with greater cognitive control (Mather and Knight 2005; Bruno et al. 2014). This presents an interesting possibility that the same individuals with greater cognitive control also have greater memory impairment. This is in line with the frontal lobe hypothesis of aging, which suggests that activity in the PFC, which underlies executive function, is increased in aging, possibly in attempts to compensate for underlying medial temporal lobe dysfunction (Cabeza et al. 1997; Cabeza and Dennis 2012). While we do not find evidence of differences between the groups on neuropsychological tests of executive function, such as LetterNumber Sequencing or Trail Making Test B, these neuropsychological tests may not be sensitive enough to detect subtle differences in cognitive control. This sheds light on the discrepancy across findings in older adults in the literature and suggests that adequate consideration for subclinical memory impairment is necessary for a more complete understanding of emotional memory alterations in aging.

Interestingly, older adults with subclinical memory impairment show preserved memory for negative and positive information, while healthy older adults show more forgetting of positive information. However, there are no differences between the groups for negative forgetting. This suggests that negative information is relatively well maintained in aging, regardless of memory impairment, while positive information shows selective trade-offs that depend on the presence of subclinical memory impairment. One possible explanation is that the positivity effect observed selectively in memory impaired older adults is associated with some of the age-associated alterations in the neurobiological processes underlying learning and memory. For example, recent data have suggested that there is an age-related shift in spatial navigational strategies and spatial memory processes from hippocampal place learning to striatal response learning (Konishi and Bohbot 2013). Importantly, individual differences showed that the older participants who used a spatial strategy to solve the task had significant activity in the hippocampus. These findings suggest that the aging process involves a shift from using the hippocampus to using the caudate nucleus during navigation, but activity in the hippocampus is sustained in a subset of healthy older adults engaged in spatial strategies.

It stands to reason that enhancement of striatal function at the expense of hippocampal function may be associated with enhanced processing of rewarding stimuli, which may mediate the observed positivity effect in our study. For example, a recent study showed that ventral striatal activity was increased in older adults when receiving reward but not during anticipation (Vink et al. 2015). This is also consistent with findings indicating that representation of reward outcome is stable in aging, compared with representation of prediction error (Samanez-Larkin et al. 2014). Thus, despite the well-documented decline in dopaminergic activity in older adults (for review, see Li and Rieckmann 2014), it is possible that at least a subset of older adults exhibit enhanced processing of rewarding information, however, this account remains speculative. Future studies should focus on the interactions between hippocampal and striatal memory systems in older adults as a function of the individual differences noted herein.

An alternative explanation is that the positivity effect observed selectively in memory impaired older adults is the result of a shift in attention to emotional satisfaction. According to the socioemotional selectivity theory (SST), when time horizons are constrained (i.e., mortality is getting closer), current goals related to emotional satisfaction are prioritized (Reed and Carstensen 2012). Older adults with subclinical memory impairment may be cognizant of some early signs of cognitive decline, and thus may shift their goals to prioritize positive information while healthy older adults do not need to shift their motivation yet. However, it is unknown whether our AI group is aware of any memory deficits. A shift to prioritizing emotional information may be due to shifts in medial prefrontal cortex (mPFC) amygdala processing, as it has been suggested that increased motivation to regulate emotion leads older adults to actively engage the mPFC differently than young adults, which may influence amygdala activity (St Jacques et al. 2010). Investigating the balance between $\mathrm{mPFC}$ and amygdala activity with an individual differences approach may offer additional clues as to the neural substrates of the positivity effect. It is worth noting that a recent study compared the positivity effect in older adults with and without Alzheimer's disease and found no differences in "time perspective," an essential facet of the SST (Bohn et al. 2016). Thus, the SST-based explanation for the selectivity of the positivity effect also remains speculative but warrants further investigation. 
There are several benefits of the study from a neuropsychological task development perspective. Since we modified a standard neuropsychological test of memory to additionally examine questions related to the processing of emotion, we retained the structure and scoring techniques that have been validated extensively in the past. The ELMT has several key advantages. First, it directly assesses and controls for emotional content, which is not possible in the original subset. Another advantage is that unlike the standard version, the modified ELMT includes a 1-wk delayed test to assess the effects of consolidation and forgetting. Given the extensive literature on how memories change over time (McGaugh 2000), a delayed test is significantly informative. Using the ELMT as a novel neuropsychological test may allow us a deeper understanding of emotional memory consolidation and forgetting in young and older adults.

A particular limitation of the study is the absence of characterization of $\mathrm{AD}$ pathology. It is possible that $\mathrm{AI}$ individuals are more likely to be those harboring $\mathrm{AD}$ pathology and thus more likely to exhibit cognitive decline in the future, however, this could not be tested in the current study. This is an ongoing topic of interest in the field (Jagust 2013) and we hope that our findings and our task may enable additional research to address these relationships.

In conclusion, the ELMT allows for the detection of subtle differences in emotional memory in older adults. Neuropsychological tests that evaluate the impact of emotion on memory will aid in a more accurate and thorough understanding of the aging brain and changes that may be associated with cognitive decline. Using these measures concomitantly with structural and functional neuroimaging of the medial temporal lobes to further understand age-related alterations in the amygdala-hippocampal network will be critical to more fully understand how emotional memory changes with age, and the association between emotion, memory, and cognitive decline.

\section{Acknowledgments}

This work was supported by NIH grants R01 MH102392, R21 AG049220, and P50 AG16573 as well as NIA training grant AG027668 support to S.L.L. (PI: M. Albert). We thank the study participants as well as Ms. Amanda Chun for assistance with recruitment and study procedures.

\section{Competing Interest Statement}

The authors declare no competing financial interests.

\section{References}

Bohn L, Kwong See ST, Fung HH. 2016. Time perspective and positivity effects in Alzheimer's disease. Psychol Aging. Epub ahead of print March $14,2016$.

Bruno D, Brown AD, Kapucu A, Marmar CR, Pomara N. 2014. Cognitive reserve and emotional stimuli in older individuals: level of education moderates the age-related positivity effect. Exp Aging Res 40: $208-223$.

Cabeza R, Dennis NA. 2012. Frontal lobes and aging: deterioration and compensation. In Principles of frontal lobe function (ed. Stuss DT, Knight RT), pp. 628-652. Oxford University Press, New York.

Cabeza R, Grady CL, Nyberg L, McIntosh AR, Tulving E, Kapur S, Jennings JM, Houle S, Craik FIM. 1997. Age-related differences in neural activity during memory encoding and retrieval: a positron emission tomography study. J. Neurosci 17: 391-400.

Craik FIM, Simon E. 1980. Age differences in memory: the roles of attention and depth of processing. In New directions in memory and aging (ed. Poon LW, Fozard JL, Cermak LS, Arenberg D, Thompson LW), pp. 95-112. Lawrence Erlbaum Associates, Hillsdale, NJ.

Estévez-González A, Kulisevsky J, Boltes A, Otermín P, García-Sánchez C. 2003. Rey verbal learning test is a useful tool for differential diagnosis in the preclinical phase of Alzheimer's disease: comparison with mild cognitive impairment and normal aging. Int J Geriatr Psychiatry 18: $1021-1028$.

Fernandes M, Ross M, Wiegand M, Schryer E. 2008. Are the memories of older adults positively biased? Psychol Aging 23: 297-306.

Gallagher M, Burwell R, Burchinal M. 1993. Severity of spatial learning impairment in aging: development of a learning index for performance in the Morris water maze. Behav Neurosci 107: 618-626.

Glisky E. 2007. Changes in cognitive function in human aging. In Brain aging: models, methods, and mechanisms (ed. Riddle D), pp. 1-10. CRC Press, Boca Raton.

Grühn D, Smith J, Baltes PB. 2005. No aging bias favoring memory for positive material: evidence from a heterogeneity-homogeneity list paradigm using emotionally toned words. Psychol Aging 20: 579-588.

Jagust W. 2013. Review vulnerable neural systems and the borderland of brain aging and neurodegeneration. Neuron 77: 219-234.

Jedynak BM, Lang A, Liu B, Katz E, Zhang Y, Wyman BT, Raunig D, Jedynak CP, Caffo B, Prince JL, et al. 2012. A computational neurodegenerative disease progression score: method and results with the Alzheimer's disease Neuroimaging Initiative cohort. Neuroimage 63: $1478-1486$.

Kensinger EA. 2008. Age differences in memory for arousing and nonarousing emotional words. J Gerontol B Psychol Sci Soc Sci 63: P13-P18.

Kensinger EA, Garoff-Eaton RJ, Schacter DL. 2007. Effects of emotion on memory specificity: memory trade-offs elicited by negative visually arousing stimuli. J Mem Lang 56: 575-591.

Koen JD, Yonelinas AP. 2014. The effects of healthy aging, amnestic mild cognitive impairment, and Alzheimer's disease on recollection and familiarity: a meta-analytic review. Neuropsychol Rev 24: $332-354$.

Konishi K, Bohbot VD. 2013. Spatial navigational strategies correlate with gray matter in the hippocampus of healthy older adults tested in a virtual maze. Front Aging Neurosci 5: 1.

Li S-C, Rieckmann A. 2014. Neuromodulation and aging: implications of aging neuronal gain control on cognition. Curr Opin Neurobiol 29: $148-158$.

Mather M, Carstensen LL. 2005. Aging and motivated cognition: the positivity effect in attention and memory. Trends Cogn Sci 9: 496-502.

Mather M, Knight M. 2005. Goal-directed memory: the role of cognitive control in older adults' emotional memory. Psychol Aging 20: $554-570$.

McGaugh JL. 2000. Memory--a century of consolidation. Science 287: 248-251.

Reagh ZM, Roberts JM, Ly M, Diprospero N, Murray E, Yassa MA. 2014. Spatial discrimination deficits as a function of mnemonic interference in aged adults with and without memory impairment. Hippocampus 24: $303-314$.

Reagh ZM, Ho HD, Leal SL, Noche JA, Chun A, Murray EA, Yassa MA. 2015 Greater loss of object than spatial mnemonic discrimination in aged adults. Hippocampus 26: 417-422.

Reed AE, Carstensen LL. 2012. The theory behind the age-related positivity effect. Front. Psychol. 3: 1-9.

Samanez-Larkin GR, Worthy DA, Mata R, McClure SM, Knutson B. 2014. Adult age differences in frontostriatal representation of prediction error but not reward outcome. Cogn Affect Behav Neurosci 14: $672-682$.

Smith TD, Adams MM, Gallagher M, Morrison JH, Rapp PR. 2000. Circuit-specific alterations in hippocampal synaptophysin immunoreactivity predict spatial learning impairment in aged rats. J Neurosci 20: 6587-6593.

Spiegel AM, Koh MT, Vogt NM, Rapp PR, Gallagher M. 2013. Hilar interneuron vulnerability distinguishes aged rats with memory impairment. J Comp Neurol 521: 3508-3523.

Stark SM, Yassa MA, Stark CEL. 2010. Individual differences in spatial pattern separation performance associated with healthy aging in humans. Learn Mem 17: 284-288.

Stark SM, Yassa MA, Lacy JW, Stark CEL. 2013. A task to assess behavioral pattern separation (BPS) in humans: Data from healthy aging and mild cognitive impairment. Neuropsychologia 51: 2442-2449.

St. Jacques P, Dolcos F, Cabeza R. 2010. Effects of aging on functional connectivity of the amygdala during negative evaluation: a network analysis of fMRI data. Neurobiol. Aging 31: 315-327.

Stranahan AM, Haberman RP, Gallagher M. 2011. Cognitive decline is associated with reduced reelin expression in the entorhinal cortex of aged rats. Cereb Cortex 21: 392-400.

Thies W, Bleiler L. 2011. Alzheimer's disease facts and figures. Alzheimer's Dement 7: 208-244. 
Vink M, Kleerekooper I, van den Wildenberg WPM, Kahn RS. 2015. Impact of aging on frontostriatal reward processing. Hum Brain Mapp 36: $2305-2317$.

Wilson IA, Ikonen S, Gureviciene I, McMahan RW, Gallagher M, Eichenbaum H, Tanila H. 2004. Cognitive aging and the hippocampus: how old rats represent new environments. J Neurosci 24: $3870-3878$

Wilson IA, Ikonen S, Gallagher M, Eichenbaum H, Tanila H. 2005. Age-associated alterations of hippocampal place cells are subregion specific. J Neurosci 25: 6877-6886.

Wong G, Dolcos S, Denkova E, Morey R, Wang L, McCarthy G, Dolcos F 2012. Brain imaging investigation of the impairing effect of emotion on cognition. J Vis Exp. doi: 10.3791/2434.

Yassa MA, Stark CEL. 2011. Pattern separation in the hippocampus. Trends Neurosci 34: 515-525.
Yassa MA, Muftuler LT, Stark CEL. 2010. Ultrahigh-resolution microstructural diffusion tensor imaging reveals perforant path degradation in aged humans in vivo. Proc Natl Acad Sci 107: 12687-12691.

Yassa MA, Lacy JW, Stark SM, Albert MS, Gallagher M, Stark CEL. 2011a. Pattern separation deficits associated with increased hippocampal CA3 and dentate gyrus activity in nondemented older adults. Hippocampus 21: $968-979$.

Yassa MA, Mattfeld AT, Stark SM, Stark CEL. 2011b. Age-related memory deficits linked to circuit-specific disruptions in the hippocampus. Proc Natl Acad Sci 108: 8873-8878.

Received February 25, 2016; accepted in revised form May 18, 2016. 


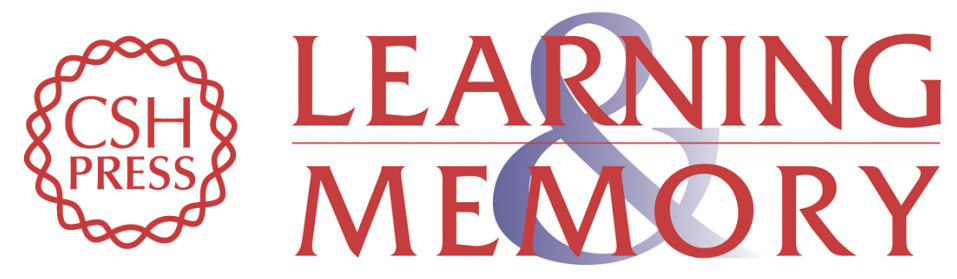

\section{Positivity effect specific to older adults with subclinical memory impairment}

Stephanie L. Leal, Jessica A. Noche, Elizabeth A. Murray, et al.

Learn. Mem. 2016, 23:

Access the most recent version at doi:10.1101/Im.042010.116

\section{Supplemental http://learnmem.cshlp.org/content/suppl/2016/07/07/23.8.415.DC1 Material}

References This article cites 35 articles, 8 of which can be accessed free at: http://learnmem.cshlp.org/content/23/8/415.full.html\#ref-list-1

Creative This article is distributed exclusively by Cold Spring Harbor Laboratory Press for the Commons first 12 months after the full-issue publication date (see

License http://learnmem.cshlp.org/site/misc/terms.xhtml). After 12 months, it is available under a Creative Commons License (Attribution-NonCommercial 4.0 International), as described at http://creativecommons.org/licenses/by-nc/4.0/.

Email Alerting Receive free email alerts when new articles cite this article - sign up in the box at the Service top right corner of the article or click here. 